\title{
DEFERRED BERNSTEIN POLYNOMIALS
}

G. G. LORENTZ

1. Introduction. If $f(x)$ is continuous in $[0,1]$, its Bernstein polynomial

$$
B_{n}^{f}(x)=\sum_{\nu=0}^{n} f(\nu / n) p_{v n}(x), \quad p_{v n}(x)=C_{n, \nu} x^{\nu}(1-x)^{n-\nu},
$$

converges to $f(x)$ uniformly in $[0,1]$. The relation $B_{n}^{f}(x) \rightarrow f(x)$ still holds for some classes of discontinuous functions (Chlodowsky [2], Herzog and Hill [3]), ${ }^{1}$ but no fairly general results can exist in this direction, since the values of $f(x)$ at rational points do not, in general, suffice to describe properly the behaviour of $f(x)$.

In this note we replace (1) by

$$
B_{n}^{f a}(x)=\sum_{v=0}^{n} f(\nu / n-a) p_{m n}(x),
$$

where $f(x)$ is supposed periodic with period 1 and $f_{a}(x)=f(x-a)$. One may expect that the values of $a$, for which (2) does not behave regularly, are exceptional. In $\$ 2$ we consider the case where $f(x)$ has one single point $c$ of unboundedness; we may assume that $c=0$. We show that in this case the behaviour of (2) depends on the degree of transcendency of $a$. We prove a gap theorem in \$3. Finally in \$4 we make some remarks about the relation of our problem to the convergence of deferred Riemann sums.

2. We need a lemma.

Lemma. Let $0<x<1$ and $\delta>0$ be fixed numbers. Then there is a $0<q<1$ and an integer $n_{0}$ such that

$$
\sum_{|v / n-x| \geqq \delta, 0 \leqq v \leqq n} p_{v n}(x) \leqq q^{n}, \quad n \geqq n_{0} .
$$

Proof. Since $n q^{n}$ is smaller than $(q+\epsilon)^{n}$ for large $n$, it is sufficient to prove the inequality for a single $p_{v n}(x)$. We may further confine our attention to $\nu$ 's such that $\nu / n$ is to the right of $x$. We have

$$
\frac{p_{\mu+1, n}(x)}{p_{\mu n}(x)}=\frac{n-\mu}{\mu+1} \frac{x}{1-x}<\left(\frac{1}{\mu / n}-1\right) /\left(\frac{1}{x}-1\right),
$$

Received by the editors October 28, 1949.

${ }^{1}$ Numbers in brackets refer to the references at the end of the paper. 
and thus for $\delta / 2 \leqq \mu / n-x<\delta$,

$$
p_{\mu+1, n}(x) / p_{\mu n}(x) \leqq\left(\frac{1}{x+\delta / 2}-1\right) /\left(\frac{1}{x}-1\right)=q_{1}<1 .
$$

There are at least $\delta n / 2-2 \operatorname{such} \mu$; and if $\mu_{0}$ is the smallest of them, for $\nu / n-x \geqq \delta$ we have

$$
p_{\nu n}(x) \leqq p_{\mu_{0} n}(x) q_{1}^{\delta n / 2-2} \leqq q^{n}, \quad 0<q<1 .
$$

Let $A(u)$ be a positive function of $u>0$. A real number $a$ admits of the approximation $A(u)$ (Koksma [5]) if there are an infinity of integers $n$ such that $|\nu / n-a| \leqq A(n)$ is fulfilled for some integer $\nu$. Almost all real $a$ do not admit of $A(u)$, if $A(u)=u^{-k}, k>2$.

THEOREM 1. Let $f(x)$ be a function with period 1 which is continuous in $0<x<1$; let

$$
|f(x)|=O\left(\exp \left(|x|^{-1 /(2+e)}\right)\right), \quad x \rightarrow 0,
$$

for some positive $\epsilon>0$. Then for almost all a

$$
\lim _{n \rightarrow \infty} B_{n}^{f_{a}}(x)=f_{a}(x)=f(x-a)
$$

holds for all $x \neq a$.

Proof. Almost all of $a$ do not admit of the approximation $u^{-(2+\epsilon / 2)}$. For any such $a$ and an $x \neq a$, choose $0<\sigma<|x-a|$ and put $|x-a|$ $-\sigma=\delta>0$. If $\bar{f}(t)$ is defined by $\bar{f}(t)=0$ for $|t-a|<\sigma$ and $\bar{f}(t)=f(t-a)$ for $|t-a| \geqq \sigma$, we have $B_{n}^{\bar{f}}(x) \rightarrow \bar{f}(x)=f(x-a)$, since $\bar{f}$ is continuous at $t=x$. Now

$$
\begin{aligned}
\left|B_{n}^{f_{a}}(x)-B_{n}^{\bar{f}}(x)\right| & \leqq \sum_{|\nu / n-a|<\sigma}|f(\nu / n-a)| p_{\nu n}(x) \\
& \leqq \sum_{|\nu / n-x|>\delta}|f(\nu / n-a)| p_{\nu n}(x) .
\end{aligned}
$$

As $|\nu / n-a| \geqq n^{-(2+e / 2)}$ for all large $n$, we obtain, by the lemma,

$$
\left|B_{n}^{f_{a}}(x)-B_{n}^{j}(x)\right| \leqq C q^{n} \exp n^{(2+\epsilon / 2) /(2+\epsilon)} \rightarrow 0, \quad n \rightarrow \infty,
$$

with some constant $C$. This proves (5).

Theorem 1 is applicable for instance to the functions $f(x)=x^{-\gamma}$, $\gamma>0$. Bernstein polynomials of the functions $(x-a)^{-\gamma}$ were also discussed by Wigert [9], who did not arrive at definitive results.

Theorem 2. If in addition to the hypotheses of Theorem 1, $f(x)$ 
tends to infinity for $x \rightarrow 0$, there is a set of the power of the continuum of the $a$ 's such that the sequence $B_{n}^{f_{a}}(x)$ is unbounded for any $x \neq a, 0,1$.

Proof. There exists a function $\psi(x)$, tending monotonically to $+\infty$ for $x \rightarrow 0$, such that $|f(x)| \geqq \psi(|x|)$. Let $\nu_{0}$ be the index for which $\left|\nu_{0} / n-a\right|<1 / 2 n$; there is at most one such $\nu_{0}$. Let $\bar{f}(t)$ be defined as in the proof of Theorem 1 , and let $\bar{B}_{n}$ be the sum obtained from $B_{n}^{f_{a}}$ by omitting the term $u$ corresponding to $\nu=\nu_{0}$. As above, we have $\bar{B}_{n} \rightarrow f(x-a)$ for $x \neq a$. Thus it is sufficient to prove that the term $u=f\left(\nu_{0} / n-a\right) p_{v_{0} n}(x)$ is unbounded. Since the smallest of the $p_{v n}(x)$ is $p_{0 n}=x^{n}$ or $p_{n n}=(1-x)^{n}$, we have

$$
|u| \geqq \psi\left(\left|\nu_{0} / n-a\right|\right) p_{v_{0} n}(x) \geqq q^{n} \psi\left(\left|\nu_{0} / n-a\right|\right), \quad 0<q<1 .
$$

The set of the $a$ 's admitting of the approximation $A(u)$ is for any positive function $A(u)$ of the power of the continuum, provided $A(u) \rightarrow 0$ for $u \rightarrow \infty$. We choose $A(u)$ such that

$$
\psi(A(n)) q^{n} \rightarrow+\infty \text {. }
$$

Then for an infinity of $n,\left|\nu_{0} / n-a\right| \leqq A(n)$, and $|u| \geqq \psi(A(n)) q^{n} \rightarrow \infty$ for these $n$, which completes the proof.

3. For the sake of completeness we mention the following theorem.

ThEOREM 3. If $f \in L_{r}, r \geqq 1$, then

$$
\begin{aligned}
& \int_{0}^{1}\left|B_{n}^{f_{a}}(x)\right|^{r} d a \leqq \int_{0}^{1}|f(a)|^{r} d a, \\
& \int_{0}^{1}\left|B_{n}^{f_{a}}(x)-f(x-a)\right|^{r} d a \rightarrow 0 \quad \text { uniformly in } x .
\end{aligned}
$$

From (6) we see that, for a fixed $x, B_{n}^{f_{a}}(x)$ is a linear operator mapping $L_{r}$ into itself with a norm less than or equal to 1 . Since $B_{n}^{f_{a}}(x)$ $\rightarrow f(x-a)$ uniformly in $x$ for any continuous $f$, (7) follows from (6) by a well known theorem on sequences of linear operators, and (6) is easily proved by using Hölder's inequality.

From Theorem 3 we see that for any integrable $f(x)$,

$$
\lim _{k \rightarrow \infty} B_{n_{k}}^{f_{a}}(x)=f(x-a)
$$

for almost all $a, x$, if $n_{k} \rightarrow \infty$ is a properly chosen sequence depending on $f$. A sequence $n_{k}$ independent of $f$ is provided by the following theorem.

THEOREM 4. Suppose that $f(x) \sim \sum_{m=-\infty}^{+\infty} c_{m} e^{2 \pi i m x}$ is the Fourier series 
of $f(x)$ in $[0,1]$ and that

$$
\sum_{m \neq 0} c_{m}^{2} \log |m|<+\infty
$$

Then (8) holds for any sequence $n_{k}$ such that

$$
n_{k+1} / n_{k} \geqq \lambda>1 \text {. }
$$

Proof. The Fourier series of $B_{n}^{f_{a}}(x)$ as a function of $a$ is

$$
B_{n}^{f a}(x)=\sum_{\nu=0}^{n} f(\nu / n-a) p_{v n}(x) \sim \sum_{m=-\infty}^{+\infty} c_{m} e^{-2 \pi i m a} \sum_{\nu=0}^{n} e^{2 \pi i m v / n} p_{\nu n}(x),
$$

and that of $B_{n}^{f_{a}}(x)-f(x-a)$ is

$$
\sum_{m=-\infty}^{+\infty} c_{m} e^{-2 \pi i m a}\left[B_{n}^{\phi_{m}}(x)-\phi_{m}(x)\right], \quad \phi_{m}(x)=e^{2 \pi i m x} .
$$

Therefore, by Parseval's identity we have

$$
\begin{aligned}
\int_{0}^{1} \int_{0}^{1} \mid B_{n}^{f a}(x)-f(x- & a)\left.\right|^{2} d a d x \\
& =\sum_{m=-\infty}^{+\infty} c_{m}^{2} \int_{0}^{1}\left|B_{n}^{\phi_{m}}(x)-\phi_{m}(x)\right|^{2} d x \\
& =\sum c_{m}^{2} \alpha_{m n}
\end{aligned}
$$

say. Since the functions $\phi_{m}(x)$ have a continuous second derivative, there is a $0 \leqq \xi \leqq 1$ such that (Bernstein [1])

$$
B_{n}^{\phi_{m}}(x)-\phi_{m}(x)=\frac{x(1-x)}{n} \phi_{m}^{\prime \prime}(\xi)
$$

and we obtain

$$
\left|B_{n}^{\phi_{m}}(x)-\phi_{m}(x)\right| \leqq C m^{2} n^{-1}
$$

with a constant $C$. We have also the trivial inequality

$$
\left|B_{n}^{\phi_{m}}(x)-\phi_{m}(x)\right| \leqq 1 .
$$

From (12) and (13) we see that

$$
\sum_{k=1}^{\infty} \alpha_{m n_{k}}=\sum_{n_{k} \leqq m^{2}}+\sum_{n_{k}>m^{2}} \leqq \sum_{n_{k} \leq m^{2}} 1+C m^{4} \sum_{n_{k}>m^{2}} n_{k}^{-2} .
$$

The first sum has no more than $\log m^{2} / \log \lambda+1=0(\log |m|)$ terms; 
and the second sum is equal to $O\left(\mathrm{~m}^{-4}\right)$. The whole sum (14) is therefore equal to $O(\log |m|)$. From (11) we see that $\sum_{k} \iint\left|B_{n_{k}}-f_{a}\right|^{2} d a d x$ converges, which completes the proof.

4. It may be worth while to remark that since $\int_{0}^{1} p_{p n}(x) d x$ $=(n+1)^{-1}$, formal integration of the relation $B_{n}^{f_{a}}(x) \rightarrow f(x-a)$ leads to

$$
\lim _{n \rightarrow \infty} \frac{1}{n+1} \sum_{\nu=0}^{n} f(\nu / n-a)=\int_{0}^{1} f(x) d x .
$$

This relation was discussed by several authors (Jessen [4], Marcinkiewicz and Salem [6], Salem [8]). The conditions on $f$ used in Theorem 4 are stronger than those under which (15) is known to hold. But for Theorem 1 the matter is just reversed, and the corresponding assertion for Riemann sums is not true even for the functions $f(x)=|x|^{-\gamma}, \gamma \geqq 1 / 2$ (Marcinkiewicz and Zygmund [7], where the case $f(x)=|x|^{-1 / 2} \log |x|$ is discussed).

\section{REFERENCES}

1. S. Bernstein, Complétement a l'article de E. Voronowskaja, C. R. (Doklady) Acad. Sci. URSS (1932) pp. 86-92.

2. I. Chlodovsky, Sur la représentation des fonctions discontinues par les polynomes de M. S. Bernstein, Fund. Math. vol. 13 (1929) pp. 62-72.

3. Fritz Herzog and J. D. Hill, The Bernstein polynomials for discontinuous functions, Amer. J. Math. vol. 68 (1946) pp. 109-124.

4. B. Jessen, On the approximation of Lebesgue integrals by Riemann sums, Ann. of Math. (2) vol. 35 (1934) pp. 248-251.

5. J. F. Koksma, Diophantische Approximationen, Berlin, 1936.

6. J. Marcinkiewicz and R. Salem, Sur les sommes riemanniennes, Compositio Math. vol. 7 (1940) pp. 376-389.

7. J. Marcinkiewicz and A. Zygmund, Mean values of trigonometric polynomials, Fund. Math. vol. 28 (1937) pp. 131-166.

8. R. Salem, Sur les sommes Riemanniennes des functions sommables, Matematisk Tidsskrift B. (1948) pp. 60-62.

9. S. Wigert, Reflexions sur le polynome d'approximations, Arkiv för Matematik Astronomi och Fysik vol. 20A, no. 5, 1927-1928, 15 pp.

UNIVERSITY OF TORONTO 